\title{
BMJ Open Observational retrospective study of the association of initial healthcare provider for new-onset low back pain with early and long-term opioid use
}

Lewis E Kazis, ${ }^{1}$ Omid Ameli, ${ }^{1,2}$ James Rothendler, ${ }^{1}$ Brigid Garrity, ${ }^{1}$ Howard Cabral, ${ }^{3}$ Christine McDonough, ${ }^{4}$ Kathleen Carey, ${ }^{1}$ Michael Stein, ${ }^{1}$ Darshak Sanghavi, ${ }^{2}$ David Elton, ${ }^{5}$ Julie Fritz, ${ }^{6}$ Robert Saper ${ }^{7}$

To cite: Kazis LE, Ameli 0 , Rothendler J, et al. Observational retrospective study of the association of initial healthcare provider for new-onset low back pain with early and longterm opioid use. BMJ Open 2019;9:e028633. doi:10.1136/ bmjopen-2018-028633

- Prepublication history and additional material for this paper are available online. To view these files, please visit the journal online (http://dx.doi. org/10.1136bmjopen-2018028633).

Received 18 December 2018 Revised 24 August 2019 Accepted 02 September 2019

A) Check for updates

(c) Author(s) (or their employer(s)) 2019. Re-use permitted under CC BY-NC. No commercial re-use. See rights and permissions. Published by BMJ.

For numbered affiliations see end of article.

Correspondence to

Dr Lewis E Kazis; lek@bu.edu

\section{ABSTRACT}

Objective This study examined the association of initial provider treatment with early and long-term opioid use in a national sample of patients with new-onset low back pain (LBP).

Design A retrospective cohort study of patients with newonset LBP from 2008 to 2013.

Setting The study evaluated outpatient and inpatient claims from patient visits, pharmacy claims and inpatient and outpatient procedures with initial providers seen for new-onset LBP.

Participants 216504 individuals aged 18 years or older across the USA who were diagnosed with new-onset LBP and were opioid-naïve were included. Participants had commercial or Medicare Advantage insurance.

Exposures The primary independent variable is type of initial healthcare provider including physicians and conservative therapists (physical therapists, chiropractors, acupuncturists).

Main outcome measures Short-term opioid use (within 30 days of the index visit) following new LBP visit and long-term opioid use (starting within 60 days of the index date and either 120 or more days' supply of opioids over 12 months, or 90 days or more supply of opioids and 10 or more opioid prescriptions over 12 months).

Results Short-term use of opioids was $22 \%$. Patients who received initial treatment from chiropractors or physical therapists had decreased odds of short-term and long-term opioid use compared with those who received initial treatment from primary care physicians (PCPs) (adjusted OR (AOR) (95\% Cl) 0.10 (0.09 to 0.10) and 0.15 (0.13 to 0.17 ), respectively). Compared with PCP visits, initial chiropractic and physical therapy also were associated with decreased odds of long-term opioid use in a propensity score matched sample (AOR $(95 \% \mathrm{Cl}) 0.21$ (0.16 to 0.27 ) and 0.29 (0.12 to 0.69$)$, respectively).

Conclusions Initial visits to chiropractors or physical therapists is associated with substantially decreased early and long-term use of opioids. Incentivising use of conservative therapists may be a strategy to reduce risks of early and long-term opioid use.
Strengths and limitations of this study

- This is a nationwide study comparing early and long-term opioid use among patients with low back pain (LBP) who seek initial care from conservative therapists, physician specialists and primary care physicians.

- We go beyond investigating the odds of opioid use for a one-time LBP event, by examining associations with both early and long-term opioid use among patients with new-onset LBP.

- We provide a broader depiction of conservative therapy than prior studies, as we included chiropractors and acupuncturists, as well as other MD specialists.

- This study assesses the impact of state regulations of access to physical therapy on choice of initial provider.

- This is a claims-based study; therefore, causation cannot be inferred, and different patient characteristics we could assess are limited.

\section{INTRODUCTION}

Over the past decade, there has been an increase in opioid use in the USA, with over 12 million Americans reporting longterm opioid use or misuse in $2015 .^{1-3}$ The National Survey on Drug Use and Health reported over 42000 prescription opioid-related deaths in 2016, with total estimated costs of prescription opioid use reaching US $\$ 78.5$ billion. ${ }^{5}$ One of the most common conditions for which opioids are prescribed is low back pain (LBP). ${ }^{2-4}$ Several studies have reported that opioids are the most frequently prescribed medication for treatment of LBP, ${ }^{45}$ and more than half of opioid users report having a history of back pain. ${ }^{6}$ This frequency of opioid prescribing is particularly concerning given that LBP is one of the three most common conditions for which Americans seek medical care. $^{27}$ 
Given the high prevalence of LBP, several guidelines have been issued for treatment, and specifically discourage opioids to treat pain. The American College of Physicians and the Centers for Disease Control recommend non-pharmacological treatments including exercise, physical therapy (PT), spinal manipulation, acupuncture and massage. ${ }^{28}$ These guidelines indicate that opioids should not be considered as a treatment option for LBP unless recommended treatments fail and if the benefits of their use outweigh the risk for the individual patient. ${ }^{28}$ Prior to the release of these recommendations, physician visits for new-onset LBP were much more common than non-pharmacological therapies like chiropractic care, PT and acupuncture. ${ }^{8-11}$

Several studies have attempted to elucidate the predictors of opioid use among patients with LBP. Comparisons of the treatment patterns of primary care physicians (PCPs) and conservative therapists (defined as chiropractors, physical therapists, acupuncturists) suggest that the use of conservative therapies for LBP may decrease the likelihood of opioid use. ${ }^{9}$ Despite these findings, there has been little research comparing early and long-term opioid use among patients seeking initial care from various providers, including PCPs, chiropractors, physical therapists and acupuncturists as well as patients seeing orthopaedic surgeons, neurosurgeons and emergency physicians. ${ }^{12-14}$ The purpose of this study is to examine the association of type of initial provider with subsequent early and long-term opioid use in a national sample of patients with new-onset LBP whose treatment could reasonably be managed by non-pharmacological therapy.

\section{METHODS}

\section{Study sample}

We conducted a retrospective study of patients seen by a healthcare provider for new-onset LBP management and who were opioid-naive at the time of the initial visit. We used de-identified administrative claims data from the OptumLabs Data Warehouse, which includes medical and pharmacy claims, laboratory results and enrolment records for commercial and Medicare Advantage (MA) enrollees. The database contains longitudinal health information on enrollees and patients, representing a diverse mixture of ages, ethnicities and geographical regions across the USA.

The various health plans individuals were enrolled in all provide comprehensive insurance coverage for physician, hospital, and prescription drug services.

The index episode of LBP was identified using claims from 2008 to 2013 with additional claims data covering 2006-2015 to ascertain pre-index visit opioid use and low back conditions and to allow a follow-up period. Patients needed to be continuously enrolled for at least 24 months both before and following the index date (total 48 months) with both medical and pharmacy claims data available during that period. The study sample included

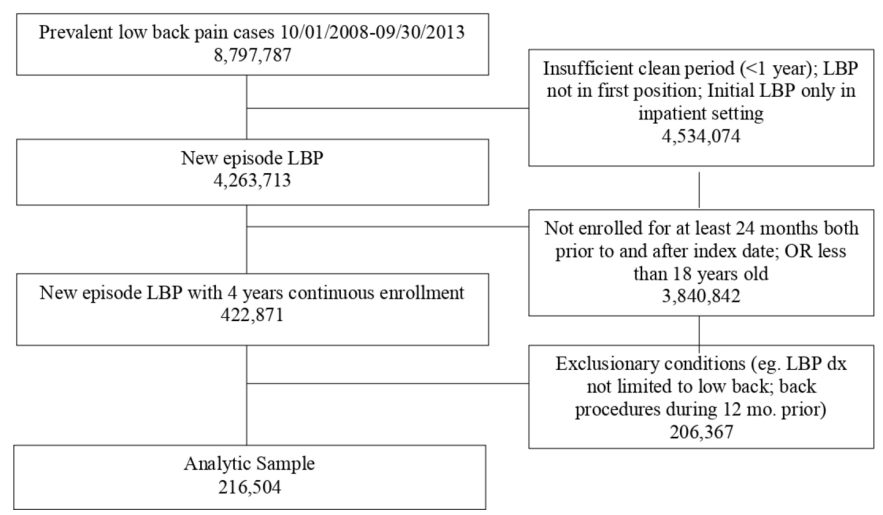

Figure 1 Inclusion and exclusion criteria. Initially, 8797787 patients with LBP were identified. Patients with an insufficient clean period (LBP within the last 1 year), patients with a diagnosis of LBP that was not in the first position of their diagnosis and LBP in only inpatient settings were excluded, reducing the number of patients to 4263713 . Patients were excluded if they were not continuously enrolled in their insurance for 24 months before and after the initial LBP visit and if they were aged $<18$ years, reducing the number of patients to 422871 . Patients with exclusionary conditions, LBP that was not limited to the low back, patients with back procedures in the 12 months prior to the index LBP visit and patients with any opioid use in the 12 months before the index visit were excluded, leaving 216504 patients in our sample.

adults aged 18 years or older with a new outpatient diagnosis of LBP who had commercial or MA insurance.

To qualify, LBP diagnosis appeared in the first location on a patient's index date insurance claim. New-onset LBP was defined as no diagnosis of LBP or back procedures, including spinal surgery, spinal injections and spinal nerve stimulators during the 12-month period prior to the index event (figure 1). Given the heterogeneity of claims data in a commercial insured population, we constructed our inclusion and exclusion criteria with the specific purpose of maximising the likelihood that our analytic sample comprised our target population.

\section{Exclusion criteria}

Patients were excluded if they had 1) a prescription for opioids in the 12 months prior to the index event (see online supplementary appendix 1 a for a list of opioids included), 2) a diagnosis of neoplasia in this 12-month period or within a 3-month period after the index LBP event or 3) a LBP-related diagnoses that would typically not be amenable to conservative therapy in the 3 months on or following the index date (see online supplementary appendix $1 \mathrm{~b}-\mathrm{d}$ for International Classification of Diseases, Ninth Revision (ICD-9) and Current Procedural Terminology (CPT) codes for inclusion/exclusion criteria). Each patient was only included in the study once.

\section{Dependent variables}

Early opioid use was defined as an opioid fill within 30 days of the index visit. Long-term use was defined as an initial 
opioid fill within 60 days of the index date and either 120 or more days' supply of opioids over 12 months, or 90 days or more supply of opioids and 10 or more opioid prescriptions over 12 months. This definition relied on previous literature. ${ }^{615}$

\section{Independent variables}

\section{Patient characteristics}

We identified patient characteristics and comorbidities using ICD-9 codes for the claims data in the 2 years prior to the index event. Characteristics included age, sex/ gender, race/ethnicity, insurance (commercial or MA) and state of residence (which was mapped to one of four US census regions: Northeast, South, Midwest and West). Race and ethnicity are defined as black, Hispanic, Asian or white (table 1). Sex/gender is determined based on enrolment records. Ethnicity was assigned by an external vendor based on a structured, rule-based system that combines analysis of first names, middle names, surnames and surname prefixes and suffixes, with geographic reference files. Values were then categorised to comply with data de-identification requirements. Physical comorbidities were assessed using a modification of the Elixhauser index ${ }^{15}$ in which mental health conditions were excluded. Other comorbidities, listed in table 2, included eight mental health conditions and a condition comprising chronic pain, fibromyalgia and fatigue. The latter conditions were included based on prior studies that have reported an association between such conditions and opioid use ${ }^{1617}$ and their specifications were based on the Centers for Medicare and Medicaid Services Chronic Conditions Data Warehouse. ${ }^{18}$

Based on the index claim date of patients with an eligible LBP diagnosis, initial providers were characterised as physical therapist, chiropractor, acupuncturist, PCP, orthopaedic surgeon, emergency medicine physician, neurosurgeon, radiologists, other non-physicians (physician assistant or nurse practitioners) or physical medicine and rehabilitation physician according to provider specialty and procedure codes. If a patient saw both a physician and a conservative therapist on the index date, the initial provider was assumed to be the physician, although this was notably a small number of individuals $(\mathrm{n}=262)$.

Patient access to PT in every state was classified as either 'limited', 'provisional' or 'unrestricted', based on the three levels of patient access outlined by the American Physical Therapy Association. ${ }^{19}$ To access PT for their initial LBP visit in limited access states, patients must have a prior relevant medical diagnosis, a recent diagnosis from a physician or other specified clinician, and/ or a prior physician referral to PT. States with provisional access permitted patients to see physical therapists with some provisions that vary by state. Restrictions in provisional access states include time and/or visit limits and physician referrals for specific interventions. Patients in unrestricted states do not face these restrictions when seeking initial care from a physical therapist. There were
6 states with limited access to PT, 26 states with provisional access and 18 states with unrestricted access to PT. ${ }^{19}$

\section{Statistical analyses}

The main analyses included multivariable logistic regressions with early opioid use and long-term opioid use as outcomes and entry-point provider as the main independent variable. The reference group for these comparisons was patients who visited PCP first for the LBP. All models were adjusted for age, sex/gender, race/ethnicity, geographic region, insurance type, the Elixhauser physical index as a continuous count of physical comorbidities and, individually, mental health comorbidities.

As a supplemental alternative to adjusting for baseline confounding through regression adjustment, we invoked two-to-one propensity score matching (two PCP: one physical therapist or two PCP: one chiropractor) without replacement to achieve baseline covariate balance among patients who initially saw chiropractor first, saw a physical therapist first or who saw PCP first. ${ }^{20} 21$ The propensity scores were calculated as predicted probabilities of chiropractor first and a physical therapist first as opposed to PCP first as a function of the following matching variables: age, sex/gender, race/ethnicity, baseline comorbidities, geographic region, calendar year of the index visit, copay, deductible, plan type, history of pregnancy within 12 months, history of vehicular accidents within 12 months, history of opioid use within the year prior to the opioidfree period, LBP diagnoses in the 13-24 months prior to the index visit and prior PT visits. The propensity score for PT also considered the state PT access category, but the chiropractor propensity score did not. We examined the covariate balance in the matched samples through the standardised mean differences of each covariate. Finally, we applied a logistic model using the matched sample to assess the association between chiropractor compared with PCP as the initial provider seen and PT compared with PCP as the initial provider seen with the outcomes early term opioid use and separately long-term opioid use (binary variables). The calliper for propensity matching was set to 0.001 for both PT and chiropractor models.

In stage 1 of the propensity analysis, multivariable odds of initial chiropractic care and initial physical therapist versus initial PCP as a function of baseline covariates were measured. Covariates measured include age, sex/gender, race, geographic region, all mental and physical comorbidities included in the previous models, pregnancy and motor vehicle accidents in the 12 months prior to the LBP visit, opioid use in the 13-24 months prior to the LBP index visit, PT, chiropractic care and acupuncture in the 24 months prior to the LBP visit and year of index visit. PT state access was only used for the PT vs PCP propensity analysis.

For all logistic models, we calculated adjusted ORs with 95\% Wald CIs. We also evaluated overall model fit, model discrimination (C-statistic) and calibration (Hosmer-Lemeshow test) for all logistic models (online supplementary appendix 2). While the Hosmer-Lemeshow models were 





Table 2 Odds of early and long-term opioid use by initial provider

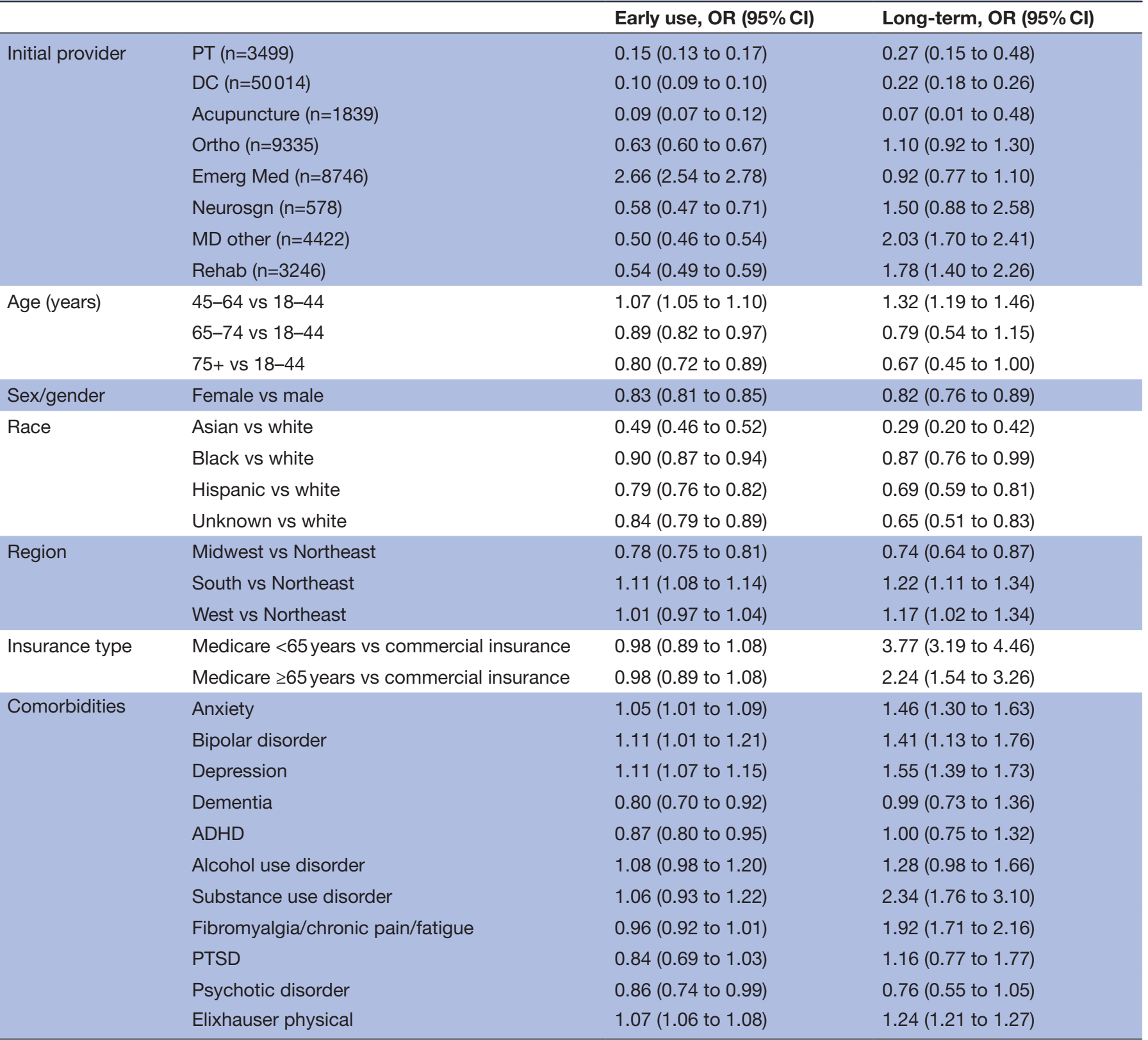

The following variables were all included in the regression: age, sex/gender, race/ethnicity, insurance, Elixhauser, which included physical comorbidities and mental health comorbidities. PCP is the reference group ( $n=114782)$; adjusted for race/ethnicity, sex/gender, region and insurance type. Two additional initial providers - other non-MD (eg, physician assistants, advance practice nurses) and radiologist were included in the analyses but not reported in this table.

${ }^{*} \mathrm{P}<0.01$.

ADHD, attention deficit hyperactivity disorder; DC, chiropractor; Emerg Med, emergency medicine physician; MD other, other physician; Neurosgn, neurosurgeon; Ortho, orthopaedic surgeon; PCP, primary care physician; PT, physical therapy; PTSD, post-traumatic stress disorder; Rehab, rehab physician.

significant, this was likely due to the large sample sizes used for this study. The differences between the observed and expected values within the decile groups was relatively small suggesting credible calibration of the models and statistical significance due to the large sample sizes. ${ }^{22} 23$ We further validated the covariate-adjusted ORs of early use and longterm use as a function of initial provider using the bootstrap method. ${ }^{24}$ The resampling rate was 200 with a two-third/ one-third sampling ratio. The bootstrapped estimates were then used to construct means and 95\% CIs for the ORs.

Patient and public involvement

No patient involvement.

\section{RESULTS}

A total of 8797787 patients had a visit with a provider for LBP during the study period, and 216504 met all 
inclusion/exclusion criteria (figure 1). More than half the patients initially saw a PCP $(n=114782,53.0 \%)$, and the most frequent initial conservative provider seen was chiropractor $(50041,23.1 \%$ ) followed by physical therapist $3499(1.6 \%)$ and acupuncturist 1839 (0.8\%). Patient demographics by initial provider are shown in table 1 . Most patients had commercial insurance (183 117, 84.7\%); the remainder had MA coverage. Of patients with an acupuncturist as the initial provider type, nearly all $(99.3 \%)$ had commercial insurance. For all other initial provider types, $87 \%-89 \%$ of patients had commercial insurance. Approximately $18 \%$ of patients received an opioid fill within 3 days of the initial LBP visit, 22\% received such a fill within the first 30 days and $1.2 \%$ met criteria for long-term use. Eighteen per cent of patients received short-acting opioids (eg, oxycodone, hydrocodone, codeine); $17.4 \%$ received prescriptions for non-steroidal anti-inflammatory drugs. The choice of initial provider varied by state PT access category. For example, in limited access states, $55.2 \%$ of initial providers were PCP, $0.9 \%$ were physical therapists and $25.7 \%$ were chiropractors. In provisional access states, the rates were $51.7 \%$ for PCPs, $1.6 \%$ for physical therapists and $23.2 \%$ for chiropractors, and in unrestricted access states, the rates were $55.8 \%$ for PCPs, $2.6 \%$ for physical therapists, and $22.6 \%$ for chiropractors.

Initial provider was associated with early and longterm opioid use outcomes in the adjusted multivariate models (table 2). Compared with seeing a PCP as initial provider, patients who first saw conservative therapists (chiropractor, acupuncturists and physical therapists) all had significantly decreased odds of both early and longterm opioid use. For early opioid use, patients initially visiting chiropractors had $90 \%$ decreased odds $(95 \%$ CI 0.09 to 0.10 ) while those visiting an acupuncturists had $91 \%$ decreased odds (95\% CI 0.07 to 0.12 ) and those visiting physical therapists had $85 \%$ decreased odds (95\% CI 0.13 to 0.17 ). Chiropractors, acupuncturists and physical therapists all had major decreased odds of longterm opioid use compared with those who initially saw PCPs $(0.22,95 \%$ CI 0.18 to 0.26 ; $0.07,95 \%$ CI 0.01 to 0.48 ; $0.27,95 \%$ CI 0.15 to 0.48 , respectively). Compared with PCPs, orthopaedic surgeons, neurosurgeons and rehab physicians as initial providers decreased patients' odds of early opioid use $(0.63,95 \%$ CI 0.60 to $0.67 ; 0.58,95 \%$ CI 0.47 to $0.71 ; 0.54,95 \%$ CI 0.49 to 0.59 , respectively), while patients seeing emergency physicians initially had significantly increased odds of early opioid use $(2.66,95 \% \mathrm{CI}$ 2.54 to 2.78). However, compared with PCP as first provider, odds for long-term opioid use were no longer significantly different for orthopaedic surgeons, neurosurgeons and emergency physicians $(1.10,95 \%$ CI 0.92 to $1.30 ; 1.50,95 \%$ CI 0.88 to $2.58 ; 0.92,95 \%$ CI 0.77 to 1.10 , respectively), but were significantly increased for rehab physicians (1.78, 95\% CI 1.40 to 2.26). (table 2). The estimates reported using bootstrapping methods indicated little or no differences with the actual results (online supplementary appendix 3 and 4 ).
Patients with anxiety, bipolar disorder and depression had significantly increased odds of long-term opioid use, as did those diagnosed with drug use disorders and fibromyalgia/chronic pain/fatigue (table 2).

Propensity score matched odds of long-term opioid use were significantly lower for chiropractic care first compared with PCP first and for PT first as compared with PCP first with ORs consistent with findings in our primary covariate-adjusted logistic models (detailed results presented in online supplementary appendix 5).

\section{Multivariable odds of initial chiropractic care and initial PT versus initial PCP as a function of baseline covariates}

Patients in states with provisional access to PT had 1.21 (95\% CI 1.05 to 1.40 ) times the odds of seeing a physical therapist initially while patients in states with unrestricted access to PT had 1.67 (95\% CI 1.41 to 1.98 ) times the odds of seeing a physical therapist initially all compared with patients in states with limited PT access (online supplementary appendix 6).

\section{DISCUSSION}

Initial treatment from conservative therapists in those with LBP was associated with a marked decrease in the odds of early and long-term opioid use. To our knowledge, this is one of the first national studies to compare early and long-term opioid use among patients with LBP who receive care from conservative therapists, physician specialists and PCPs. Although the impact of unmeasured confounders cannot be ruled out in this retrospective observational cohort study, the findings warrant careful consideration.

Several factors may help explain the apparent protective effect of conservative therapists. Since non-physicians are unable to prescribe opioids, patients seeking conservative therapy do not receive opioid prescriptions at the index visit, and subsequent visits to an MD would be required to obtain such prescriptions. There may be selection bias among patients choosing to seek initial treatment from conservative therapists, and such biases could be related to educational level or preferences which may also result in decreased desire for those patients to use opioids. Additionally, the conservative therapy provided may result in decreased pain and improved back-related function so that patients do not need or seek opioid medications. A growing body of evidence suggest that spinal manipulation, massage, acupuncture and superficial heat are effective for reducing acute LBP intensity and improving function. ${ }^{23} 25$ The conservative therapists studied in this analysis can incorporate one or more of these approaches: physical therapist (manipulation, massage, heat), chiropractor (manipulation, massage, heat) and acupuncturist (acupuncture, massage). ${ }^{23}$ Therefore and importantly, early engagement of conservative therapists may decrease initial opioid prescriptions in association with MD visits by providing the opportunity to incorporate evidence-based non-pharmacological interventions. 
Notably, state access to PT had a considerable association with choice of initial provider. Compared with states with limited access to PT, patients in states with unrestricted and provisional access had $67 \%$ and $21 \%$ higher odds of visiting physical therapists initially, respectively. Given that initial PT is associated with significant reductions in early and long-term opioid use, these observations are potentially important.

The discrepancy between early and long-term use among PCPs and physicians specialists is also interesting. While patients who initially see orthopaedic surgeons, neurosurgeons, rehabilitation physicians and other physicians have significantly lower odds of early opioid use compared with PCPs, patients who initially see these physicians have similar or increased odds of long-term opioid use compared with PCPs. While we do not have a measure of pain severity, we hypothesise that physician specialists are trying to avoid prescribing opioids at the index visit, but if patients return at follow-up due to persistent pain, physicians are likely to prescribe opioids.

\section{Comparisons to prior studies}

Our results are consistent with prior studies showing high rates of opioid prescription fills for patients with LBP who were seen by emergency department physicians. ${ }^{3}{ }^{26}$ While there are very few studies suggesting that PT and chiropractic care are used in emergency departments in the USA, ${ }^{27}$ other countries have successfully introduced physical therapists into emergency departments to treat patients with LBP. ${ }^{27-29}$

Certain comorbidities were also associated with increased odds of opioid use. Patients with fibromyalgia, chronic pain and fatigue and those with certain psychiatric conditions, including anxiety, bipolar disorder and depression had greater odds of long-term opioid use than patients without these disorders. This is consistent with recent evidence suggesting that adults with mental health conditions account for half of opioid prescriptions in the USA. $^{30}$

A recent study by Frogner $e t$ al also found that patients with LBP who saw physical therapists initially had lower opioid use, although this study only focused on six states. ${ }^{13}$ Another recent study by Hayward et al evaluated the use of non-opioid treatments from various providers, including physical therapists and physicians, however this was a descriptive study across only 16 states. ${ }^{31}$ Our study examines the association of conservative therapy on opioid use with a sample that is national in scope. We also provide a broader depiction of conservative therapy, as we included chiropractors and acupuncturists in addition to physical therapists, as well as other MD specialists. Importantly, we find that conservative therapists other than physical therapists, including chiropractors and acupuncturists, when seen first after a new episode of LBP are apparently protective of early and long-term use of opioids when compared with PCPs. Finally, we go beyond investigating the odds of opioid use for a one-time LBP event, by examining associations with both early and long-term opioid use among patients with new-onset LBP, using rigorous definitions of recent onset.

Fritz et al found that early PT was associated with decreased opioid prescriptions, ${ }^{32}$ and a review by Ojha $e t$ al noted that early PT as treatment for musculoskeletal disorders was associated with decreased frequency of opioid prescriptions, although this was based on a limited number of outcomes. ${ }^{33}$ Thackerary et al also suggested that the odds of receiving an opioid prescription among Medicaid beneficiaries were reduced for those who had a PT consult, as compared with those who did not. ${ }^{34}$ While these studies did not measure the odds of opioid use among patients who initially saw physical therapists compared with other initial providers, our results are consistent with previously published reports that PT is associated with lower odds of opioid use.

\section{Limitations}

There are several limitations to this study. Analysis was conducted using claims data, limiting our generalisability beyond commercial and MA enrolled patients. However, the sample is national in scope and provides a range of sociodemographic and clinical characteristics. The observational nature of the study and the use of claims data limited our ability to eliminate the influence of unmeasured confounders. In particular, confounding by indication may bias our results. Patients who seek early treatment with non-pharmacological practitioners may be more likely to have mild back pain that does not require more aggressive treatment such as opioids, compared with individuals with more severe back pain who may be more likely to see practitioners able to prescribe opioids. Studies have suggested that those with more severe LBP are more likely to receive opioids, ${ }^{35}$ and if patients with less severe pain were more likely to choose conservative therapists rather than physicians, this could contribute to overestimation of the protective effect of conservative therapy on opioid use. Several prior studies have shown comparable baseline pain scores for those who choose conservative therapists compared with those who choose to see physicians initially, however it is important to note that these studies had different patient populations than this study as they were conducted either only in one state or in countries other than the USA. ${ }^{35-39}$ Other unmeasured confounders may include patient preferences and behavioural characteristics. For example, those who chose conservative therapists as initial providers for LBP may have preferences to avoid pharmacological and/or opioid therapy. Therefore, while the associations between initial healthcare providers for LBP and subsequent opioid therapy found in our analyses have potentially important implications, one cannot infer causality due to the observational retrospective nature of the study.

\section{Policy implications and future research}

In conclusion, our results suggest that use of conservative therapists as initial providers for new-onset LBP are associated with lower odds of early and long-term use of 
opioids compared with PCPs. Further research in other settings and prospective pragmatic trials will be useful to confirm our findings and to better understand other factors that influence choice of initial providers for LBP. Future research should include personal factors such as preferences related to opioid use, and medical, non-medical and specialists as initial providers. Factors related to health plan benefit design such as out-of-pocket costs for treatment alternatives (eg, PT and chiropractor visits, opioids) and impact of gatekeeper requirements on care patterns should be investigated.

\section{Author affiliations}

'Department of Health Law, Policy and Management, Boston University School of Public Health, Boston, Massachusetts, USA

${ }^{2}$ OptumLabs, Cambridge, Massachusetts, USA

${ }^{3}$ Department of Biostatistics, Boston University School of Public Health, Boston, Massachusetts, USA

${ }^{4}$ University of Pittsburgh School of Health and Rehabilitation Sciences, Pittsburgh, Pennsylvania, USA

${ }^{5}$ OptumHealth, Eden Prairie, Minnesota, USA

${ }^{6}$ Physical Therapy and Athletic Training, University of Utah, Salt Lake City, Utah, USA ${ }^{7}$ Boston University Medical Campus, Boston, Massachusetts, USA

Acknowledgements The authors would like to thank members of the OptumLabs steering committee for their feedback and advice on this project. The authors would like to thank Henry Henk, PhD, OptumLabs and Pamela C. Hansen, PhD, OptumLabs.

Contributors LEK, JR, OA, HC, CMCD, KC and RS were responsible for project design and conceptualisation. $\mathrm{OA}$ and $\mathrm{HC}$ were responsible for data analysis. LEK, JR, OA, HC, CMcD, KC, RS, MS and BG were responsible for writing of the manuscript. DS, DE and JF provided critical feedback during the analysis and writing of the manuscript. All authors were involved in the editing and review of the manuscript.

Funding This project was funded by the American Physical Therapy Association, OptumLabs and UnitedHealthCare.

Disclaimer The American Physical Therapy Association, OptumLabs and UnitedHealthCare had no editorial control or influence for the final content of this paper.

Competing interests Drs OA, DS and DE work for OptumLabs but do not have any competing interests to report. This study was commissioned by OptumLabs and the American Physical Therapy Association (APTA). The funders provided comments on the production of the manuscript. The primary author, Dr LEK Professor at Boston University, had final editorial comment on the manuscript.

Patient consent for publication Not required.

Ethics approval This study was approved by Boston Medical Center (IRB approval number: $\mathrm{H}-36499)$.

Provenance and peer review Not commissioned; externally peer reviewed.

Data availability statement Certain data from OptumLabs Data Warehouse may be available upon request.

Open access This is an open access article distributed in accordance with the Creative Commons Attribution Non Commercial (CC BY-NC 4.0) license, which permits others to distribute, remix, adapt, build upon this work non-commercially, and license their derivative works on different terms, provided the original work is properly cited, appropriate credit is given, any changes made indicated, and the use is non-commercial. See: http://creativecommons.org/licenses/by-nc/4.0/.

\section{REFERENCES}

1. Hughes A, Williams M, Lipari R. Prescription drug use and misuse in the United States: results from the 2015 national survey on drug use and health, 2016.

2. Dowell D, Haegerich TM, Chou R. CDC guideline for prescribing opioids for chronic pain--United States, 2016. JAMA 2016;315.
3. Shmagel A, Ngo L, Ensrud K, et al. Prescription medication use among community-based U.S. adults with chronic low back pain: a cross-sectional population based study. J Pain 2018;19:1104-12.

4. Opioid Overdose [Internet]. Centers for disease control and prevention. centers for disease control and prevention, 2017. Available: https://www.cdc.gov/drugoverdose/data/statedeaths.html [Accessed cited 6 Nov 2018].

5. Gore M, Tai K-S, Sadosky A, et al. Use and costs of prescription medications and alternative treatments in patients with osteoarthritis and chronic low back pain in community-based settings. Pain Practice 2012;12:550-60.

6. Boudreau D, Von Korff M, Rutter CM, et al. Trends in De-facto longterm opioid therapy for chronic non-cancer pain. Pharmacoepidemiol Drug Saf 2009;18:1166-75.

7. Licciardone JC. The epidemiology and medical management of low back pain during ambulatory medical care visits in the United States. Osteopath Med Prim Care 2008;2:11.

8. Qaseem A, Wilt TJ, McLean RM, et al. Noninvasive treatments for acute, subacute, and chronic low back pain. Ann Intern Med 2017; 166 .

9. Fritz JM, Kim J, Dorius J. Importance of the type of provider seen to begin health care for a new episode low back pain: associations with future utilization and costs. J Eval Clin Pract 2016;22:247-52.

10. Cherkin DC, Deyo RA, Wheeler K, et al. Physician variation in diagnostic testing for low back pain. who you see is what you get. Arthritis Rheum 1994;37:15-22.

11. Webster BS, Cifuentes M, Verma S, et al. Geographic variation in opioid prescribing for acute, work-related, low back pain and associated factors: a multilevel analysis. Am J Ind Med 2009;52:162-71.

12. Fritz JM, Cleland JA, Speckman M, et al. Physical therapy for acute low back pain. Spine 2008;33:1800-5.

13. Frogner BK, Harwood K, Andrilla CHA, et al. Physical therapy as the first point of care to treat low back pain: an instrumental variables approach to estimate impact on opioid prescription, health care utilization, and costs. Health Serv Res 2018;53:4629-46.

14. Martin BC, Fan M-Y, Edlund MJ, et al. Long-Term chronic opioid therapy discontinuation rates from the TROUP study. J Gen Intern Med 2011;26:1450-7.

15. Elixhauser A, Steiner C, Harris DR, et al. Comorbidity measures for use with administrative data. Med Care 1998;36:8-27.

16. Feingold D, Brill S, Goor-Aryeh I, et al. The association between severity of depression and prescription opioid misuse among chronic pain patients with and without anxiety: a cross-sectional study. $J$ Affect Disord 2018;235:293-302.

17. Goesling J, Henry MJ, Moser SE, et al. Symptoms of depression are associated with opioid use regardless of pain severity and physical functioning among treatment-seeking patients with chronic pain. $J$ Pain 2015;16:844-51.

18. Chronic Condition Data Warehouse. Centers for Medicare and Medicais services. Available: https://www.ccwdata.org/web/guest/ condition-categories

19. Levels of Patient Access to Physical Therapist Services in the States [Internet]. American physical therapy association, 2016. Available: https://www.apta.org/uploadedFiles/APTAorg/Advocacy/State/ Issues/Direct_Access/DirectAccessbyState.pdf

20. Stuart EA, King G, Imai K, et al. Matchlt: nonparametric preprocessing for parametric causal inference. J Stat Softw 2011;42.

21. Dehejia RH, Wahba S. Propensity score-matching methods for nonexperimental causal studies. Rev Econ Stat 2002;84:151-61.

22. Feudtner $\mathrm{C}$, Hexem KR, Shabbout M, et al. Prediction of pediatric death in the year after hospitalization: a population-level retrospective cohort study. J Palliat Med 2009;12:160-9.

23. Chou R, Deyo R, Friedly J, et al. Nonpharmacologic therapies for low back pain: a systematic review for an American College of physicians clinical practice guideline. Ann Intern Med 2017;166:493-505.

24. Jackknife and bootstrap analyses, 2010. Available: http://support. sas.com/kb/24/982.html [Accessed cited 6 Nov 2018].

25. Chou R, Huffman LH. Nonpharmacologic therapies for acute and chronic low back pain: a review of the evidence for an American pain Society/American College of physicians clinical practice guideline. Ann Intern Med 2007;147:492.

26. Cantrill SV, Brown MD, Carlisle RJ, et al. Clinical policy: critical issues in the prescribing of opioids for adult patients in the emergency department. Ann Emerg Med 2012;60:499-525.

27. Lebec MT, Jogodka CE. The physical therapist as a musculoskeletal specialist in the emergency department. J Orthop Sports Phys Ther 2009;39:221-9.

28. Lau PM-Y, Chow DH-K, Pope MH. Early physiotherapy intervention in an accident and emergency department reduces pain and improves 
satisfaction for patients with acute low back pain: a randomised trial. Aust J Physiother 2008;54:243-9.

29. Fleming-McDonnell D, Czuppon S, Deusinger SS, et al. Physical therapy in the emergency department: development of a novel practice venue. Phys Ther 2010;90:420-6.

30. Davis MA, Lin LA, Liu H, et al. Prescription opioid use among adults with mental health disorders in the United States. J Am Board Fam Med 2017;30:407-17.

31. Heyward J, Jones CM, Compton WM, et al. Coverage of nonpharmacologic treatments for low back pain among US public and private insurers. JAMA Network Open 2018;1.

32. Fritz JM, Childs JD, Wainner RS, et al. Primary care referral of patients with low back pain to physical therapy: impact on future health care utilization and costs. Spine 2012;37:2114-212.

33. Ojha HA, Wyrsta NJ, Davenport TE, et al. Timing of physical therapy initiation for nonsurgical management of musculoskeletal disorders and effects on patient outcomes: a systematic review. J Orthop Sports Phys Ther 2016;46:56-70.

34. Thackeray A, Hess R, Dorius J, et al. Relationship of opioid prescriptions to physical therapy referral and participation for
Medicaid patients with new-onset low back pain. J Am Board Fam Med 2017;30:784-94.

35. Larson MJ, Adams RS, Ritter GA, et al. Associations of early treatments for low-back pain with military readiness outcomes. $J$ Altern Complement Med 2018;24:666-76.

36. Carey TS, Evans AT, Hadler NM, et al. Acute severe low back pain. Spine 1996;21:339-44.

37. Carey TS, Garrett J, Jackman A, et al. The outcomes and costs of care for acute low back pain among patients seen by primary care practitioners, chiropractors, and orthopedic surgeons. The North Carolina back pain project. N Engl J Med 1995;333:913-7.

38. Houweling TAW, Braga AV, Hausheer T, et al. First-contact care with a medical vs chiropractic provider after consultation with a Swiss telemedicine provider: comparison of outcomes, patient satisfaction, and health care costs in spinal, hip, and shoulder pain patients. $J$ Manipulative Physiol Ther 2015;38:477-83.

39. Walker BF, Muller R, Grant WD. Low back pain in Australian adults. health provider utilization and care seeking. J Manipulative Physiol Ther 2004;27:327-35. 
Correction: Observational retrospective study of the association of initial healthcare provider for new-onset low back pain with early and long-term opioid use

Kazis LE, Ameli O, Rothendler J, et al. Observational retrospective study of the association of initial healthcare provider for new-onset low back pain with early and long-term opioid use. BMJ Open 2019;9:e028633. doi: 10.1136/bmjopen-2018-028633

The following amendments in Abstract were considered to the original version of this article.

1. Acupuncturists have been included, along with chiropractors and physical therapists, as one of the 3 types of conservative therapists that were included in the analyses.

2. Referring to opioid use, the term 'early' rather than 'short-term' is now used throughout the abstract, consistent with the terminology in the full manuscript.

3. In the 'Main outcomes measures' section, the wording has been slightly modified to clarify the outcomes of interest.

4. In the 'Results' section, the odds ratios for opioid use have been clarified and expanded. In the original abstract, the odds ratios for early opioid use were provided for chiropractors and physical therapists based on the multivariate analyses (from Table 2 of the manuscript), but they were not clearly labelled. The odds ratios for long-term opioid use were provided for chiropractors and physical therapists based on the propensity analyses (Appendix Table 5). In the revised abstract, the odds ratios for both the early and long-term use of opioids are more clearly labelled, both are based on multivariate analyses, and the results for acupuncturists are now provided in addition to those for chiropractors and physical therapists.

5. In the 'Conclusion' section, the phrase 'compared with PCPs' was added to clarify that the comparison was to PCPs.

The text of the revised abstract follows:

Objective This study examined the association of initial provider treatment with early and long-term opioid use in a national sample of patients with new-onset low back pain (LBP).

Design A retrospective cohort study of patients with new-onset LBP from 2008 to 2013. Setting The study evaluated outpatient and inpatient claims from patient visits, pharmacy claims and inpatient and outpatient procedures with initial providers seen for new-onset LBP.

Participants 216504 individuals aged 18 years or older across the USA who were diagnosed with new-onset LBP and were opioid-naïve were included. Participants had commercial or Medicare Advantage insurance.

Exposures The primary independent variable is type of initial healthcare provider including physicians and conservative therapists (chiropractors, physical therapists and acupuncturists).

Main outcome measures Early opioid use (within 30 days of the index visit for newonset LBP) and long-term opioid use (starting within 60 days of the index visit and either 120 or more days' supply of opioids over 12 months, or 90 days or more supply of opioids and 10 or more opioid prescriptions over 12 months).

Results Early use of opioids was 22\%. Patients who received initial treatment from chiropractors, physical therapists and acupuncturists had decreased odds of early opioid use compared with those who received initial treatment from primary care physicians (PCPs) (adjusted OR (AOR) (95\% CI) 0.10 (0.09 to 0.10), 0.15 (0.13 to 0.17 ) and 0.09 (0.07 to 0.12), respectively). Compared with PCP visits, initial chiropractic, physical therapy and acupuncture also were associated with decreased odds of long-term opioid use (AOR (95\% CI) 0.22 (0.18 to 0.26$), 0.27$ (0.15 to 0.48$)$, and 0.07 (0.01 to 0.48$)$, respectively). 
Conclusions Initial visits to chiropractors, physical therapists or acupuncturists, compared with PCPs, are associated with substantially decreased early and long-term use of opioids. Incentivizing use of conservative therapists may be a strategy to reduce risks of early and long-term opioid use.

Open access This is an open access article distributed in accordance with the Creative Commons Attribution Non Commercial (CC BY-NC 4.0) license, which permits others to distribute, remix, adapt, build upon this work non-commercially, and license their derivative works on different terms, provided the original work is properly cited, appropriate credit is given, any changes made indicated, and the use is non-commercial. See: http://creativecommons.org/licenses/by-nc/4.0/.

C Author(s) (or their employer(s)) 2020. Re-use permitted under CC BY-NC. No commercial re-use. See rights and permissions. Published by BMJ.

BMJ Open 2020;10:e028633corr1. doi:10.1136/bmjopen-2018-028633corr1

(D) Check for updates 